\title{
Desarrollo de las competencias digitales de docentes a través de programas de intervención 2020
}

\section{Role of the speech therapy cabinet in the educational inclusion of young university students with a diagnosis of stuttering}

\author{
Sandra Úrsula Benavente-Vera \\ Universidad César Vallejo, Lima, Perú \\ ORCID: https://orcid.org/0000-0001-5954-4553 \\ Miriam Liliana Flores Coronado \\ Universidad Nacional Federico Villarreal, Lima, Perú \\ Orcid: https://orcid.org/0000-0003-4409-585X \\ Felipe Guizado Oscco \\ Universidad César Vallejo, Lima, Perú \\ Orcid: https://orcid.org/0000-0003-3765-7391

\section{Luis Alberto Núñez Lira} \\ Universidad Nacional Mayor de San Marcos, Lima, Perú \\ ORCID: https://orcid.org/0000-0003-3542-9117
}

*Correspondence

Email:1nunezl@unmsm.edu.pe
Cite as:

Benavente-Vera, S; Flores, M; Guizado, F; Núñez, L. (2021). Desarrollo de las competencias digitales de docentes a través de programas de intervención 2020. Propósitos y Representaciones, $\quad 9(1), \quad$ e1034. Doi: http://dx.doi.org/10.20511/pyr2021.v9n1.1034

(C) Universidad San Ignacio de Loyola, Vicerrectorado de Investigación, 2021

(c) BY-NC-ND This article is distributed under license CC BY-NC-ND 4.0 International (http://creativecommons.org/licenses/by-nc-nd/4.0/) 


\section{Resumen}

La investigación titulada Programa CODI para el desarrollo de las competencias digitales de docentes de la I.E. N 1129, La Victoria, 2020, tuvo como finalidad demostrar la eficacia de su aplicación en el desarrollo de las competencias digitales, para lo cual utilizó una metodología cuantitativa de diseño experimental con cuatro tratamientos y se empleó una población conformada por los docentes de la institución educativa. Los análisis de confiabilidad de los datos recogidos con los instrumentos validados permitieron demostrar su confiabilidad a niveles altos, datos que se aplicaron a una muestra piloto. La estadística aplicada fue de Anova de un factor que permitió obtener resultados luego de realizado el proceso de inducción y capacitación ha permitido demostrar la efectividad del programa CODI en el desarrollo de las competencias digitales de docentes de la I.E. № 1129, La Victoria, 2020, con la aplicación de cuatro tratamientos en un diseño experimental, y que el análisis estadístico empleado señala el crecimiento de su aprendizaje.

Palabras clave: competencias docentes, formación de docentes, taller educativo, tecnología de la información

\section{Summary}

The research entitled CODI Program for the development of digital competences of teachers of the I.E. $N^{\circ} 1129$, La Victoria, 2020, aimed to demonstrate the effectiveness of its application in the development of digital skills, for which it used a quantitative methodology of experimental design with four treatments and a population made up of teachers from the educational institution. The reliability analyzes of the data collected with the validated instruments made it possible to demonstrate its reliability at high levels, data that were applied to a pilot sample. The applied statistics was from Anova of a factor that allowed to obtain results after the induction and training process has been carried out, which has allowed to demonstrate the effectiveness of the CODI program in the development of the digital competences of teachers of the I.E. $N{ }^{\circ} 1129$, La Victoria, 2020, with the application of four treatments in an experimental design, and that the statistical analysis used indicates the growth of their learning.

Keywords: teaching skills, teacher training, educational workshop, information technology 


\section{Introducción}

El siglo XXI llegó para transformar el mundo con el uso de la tecnología, no hay persona que no haga uso de un medio tecnológico, haciéndose indispensable recibir una formación que nos permita ser competentes digitales. Los países desarrollados ya han asumido una educación basada en el uso continuo de las Tics donde su uso ponderado de los docentes depende de las habilidades que estos desarrollen para ponerlos en práctica en su quehacer educativo. Las escuelas del mundo se han ido implementando para desenvolverse en entornos virtuales, priorizando la preparación del docente, para que sean capaces de incluir herramientas tecnológicas en las actividades de aprendizaje.

La transformación educativa es la forma como hemos recibido la alfabetización, pues hace algún tiempo era considerada una persona alfabetizada alguien que tenía la capacidad de leer, comprender, comentar, escribir, respetando la ortografía y caligrafía con un vocabulario adecuado; en la actualidad no solo deber hacer todo lo mencionado anteriormente, sino que además debe tener cierta competencia tecnológica y digital que le permita desenvolverse frente a una computadora y poder navegar usando diferentes sitios web; además, discriminar la información que se encuentra en la red, usar un correo, participar en un foro, entre otras actividades virtuales (Area \& Guarro, 2012)

En el Perú, a consecuencia del COVID 19, se inicia la educación a distancia en su totalidad del sistema, exponiendo las debilidades de los docentes debido a las carencias en el uso de la tecnología. Adicionalmente a ello, las instituciones no han implementado acciones para favorecer este conocimiento. En estudios previos se ha podido determinar que la educación a distancia es un sistema o un método educativo que no requiere de la presencia del alumno, con la intervención de tecnologías diversas, ocurriendo en un lugar diferente, requiriendo de la tecnología y organización institucional. Asimismo, transforma los tradicionales esquemas en el desarrollo de enseñanza-aprendizaje, tanto para el estudiante como para el docente, existiendo una característica, que la educación a distancia se da en una población poco numerosa, además es flexible, dinámica y se adapta al medio (Juca, 2016)

La tecnología digital permite el uso de los recursos de comunicación comunitaria y se le considera educativo en tanto influyan sobre en lo que aprenden los niños, cuya innovación ha tenido un rápido crecimiento, y no ha sido aprovechado ni por las instituciones ni docentes. Sin embargo, no estuvo listo el sistema educativo, y por tanto hubo muchas dificultades para asumir los nuevos retos evidenciando en algunos la 
precariedad de la formación de los docentes en el uso y manejo de las TIC (Moral \& Villalustre, 2010)

El desconocimiento del manejo de la tecnología limita al docente a simplemente dejar tareas, fichas de trabajo, que los estudiantes tienen que desarrollar en casa, demostrando el uso estrategias tradicionales para el logro de competencias, lo que supone un gran reto en el escenario actual. Las clases virtuales del otorgados por el Ministerio de Educación, no cubre las expectativas de niños ni padres, estableciéndose la necesidad de que el maestro esté preparado para elaborar materiales digitales, que sirvan de soporte a sus estudiantes, ya que los padres de familia no están preparados para enseñar y si a ello le sumamos que en algunos hogares hay varios hijos trabajando en casa, el problema se acrecienta. Ante este escenario nos planteamos como problema ¿Qué efectos se tienen la aplicación de un programa de intervención en el desarrollo de las competencias digitales docentes?

Las investigaciones precedentes como los de (Ojeda, Reyes, \& Reyes, 2018) y (López, 2018) han hecho contribuciones importantes en desarrollar programas de alfabetización digital para profesores de escuelas normales donde se demuestran el logro de resultados satisfactorios como también la necesidad de la actualización permanente por parte del profesorado. Por su parte, (Chou, Valdés, \& Sánchez, 2017; Pérez, Jordano de la Torre, \& Martín-Cuadrado, 2017) en la aplicación de sus programas logran resultados exitosos con referencia a la innovación y perfeccionamiento docente adquiriendo nuestras estrategias didácticas. Además, la importancia del trabajo de (Hernández, Gámiz, \& Romero, 2020) donde indagaron la transformación de la competencia digital en docentes universitarios de México y España concluyendo con una propuesta de indicadores para cada categoría, que permiten entender la competencia digital, como una transformación y como un suplemento para planes e instrucción del profesor.

En estudios correlacionales con variables orientadas al desarrollo profesional del docente y competencias digitales encontraron competencias positivas y de niveles intermedios y fuertes (Guizado, Menacho, \& Salvatierra, 2019; Lamacponga, 2019; Hatlevik, 2017)

Otros estudios demuestran las debilidades de los docentes en cuanto a su preparación digital, teniendo grandes dificultades en la realización de los procesos de aprendizaje como los de (Lores, Sánchez, \& García, 2019; Martínez, El Berdai, \& Ortiz, 2017) 
Con referencia a la variable de estudio, esta basada en el análisis de tres modelos teóricos: la teoría educativa llamada Conectivismo de Siemens, la cual aparece en esta era digital y se vale de las nuevas tecnologías para su aplicación y descripción. En el año 2004 Siemens introduce el término y lo analiza para darle una estructura, es así que menciona que el conectivismo muestra un modelo de aprendizaje que requiere de ciertas habilidades de aprendizaje que van de la mano con acciones que permitan que las personas se desenvuelvan en un ambiente digital, en el cual el aprendizaje no es considerado como una actividad individual, sino más bien, colectiva, en la cual las redes permiten el intercambio fluido de conocimientos y el trabajo cooperativo. Siemens, también afirma que siempre surge nuevos conocimientos y es por ello que el docente debe estar en permanente preparación, para ello no solo debe conocer las herramientas web, sino también mantenerse actualizado.

El conectivismo se basa en las habilidades de aprendizaje y las funciones necesarias para que las personas utilicen las herramientas informáticas, en las cuales el aprendizaje se construye gracias al proceso generado por el estudiante y en el cual el docente guía y conduce el mismo, en todo caso la función del docente se ve alterada, pues es el que acompaña, guía, conduce, realiza la tutoría para el trabajo en redes (Siemens, 2004)

La teoría de Siemens está basada en principios: aprendizaje y el conocimiento se definen desde diferentes perspectivas, basado en diversas fuentes de información. Para el aprendizaje haciendo uso de la tecnología se abre un campo extenso para descubrir mucho más, se aprende cuando hay conexiones, donde mantener el conocimiento actualizado es la finalidad del aprendizaje conectivista y lo que se aprende en el presente es válido, pero susceptible a modificaciones de acuerdo al contexto. Por ello, el conectivismo se trata de la aplicación de los principios de redes para definir tanto el conocimiento como el mismo proceso de aprendizaje (Kenneth, 2012).

Como segunda teoría de aprendizaje, la corriente constructivista y el constructivismo social (Ortega \& Gacitúa, 2008) manifestando que la interacción social se potencia a través del uso de los medios tecnológicos, ya que permite que el aprendizaje se dé haciendo uso de los medios y ello permite que todos los participantes puedan aprender de manera conjunta. Consiste en darle relevancia a todo aquel espacio en el que se pueda construir conocimiento gracias al intercambio permanente entre estudiantes y docentes y en este medio es fundamental la toma de conciencia del papel que desempeña cada uno de los protagonistas de este espacio, pues cuando se toma 
conciencia real de las posibilidades, como también desafíos que implica esta forma de enseñanza aprendizaje se obtienen resultados satisfactorios.

Para (Piaget, 1978) el proceso de aprendizaje es la experiencia directa y asimilación de la información que son fundamentales, así también los errores y el encontrar soluciones. Se marca dos puntos referentes al constructivismo, como primer aspecto, se considera fundamental la interacción de las personas o estudiantes con el medio real, con el contexto o la situación, como segundo aspecto menciona que las personas con un elemento activo dentro del medio en el que se encuentren, pues brindan significado con sus aportaciones, ideas, pensamientos, como también, reciben las ideas, representaciones y pensamientos de las otras personas que conforman el medio de intercambio, ello ocurre porque se comparten entornos cognitivos. Lo mencionado líneas arriba es lo que ocurre al hacer uso de las diferentes herramientas tecnológicas, pues existe el intercambio y la devolución de parte de los miembros que conforman una comunidad virtual, pues se busca que los estudiantes investiguen, compartan e intercambien conocimientos para enriquecer el aprendizaje colectivo.

Con referencia a la teoría socio cultural de Vygotsky, la cual plantea que el medio social es fundamental para el desarrollo de la persona. La perspectiva sociocultural tiene una visión del aprendizaje en la que el entorno social y la colaboración mediada por el lenguaje como herramienta psicológica juegan un papel fundamental para el logro de objetivos. Para Vygotsky, las actividades humanas ocurren en sociedad y por ellos no se pueden comprender fuera de ellas, ello quiere decir que los procesos mentales se dan en la medida que se realicen interacciones con las demás personas. Según su teoría, es fundamental establecer diálogos cooperativos, en los cuales las personas generen aprendizaje en comunidad y con interacción de los participantes.

En definitiva, al hablar de la teoría sociocultural y su repercusión, no cabe duda de que, al hacer uso de las redes, intercambiar información, producir nueva información y aprender de forma colaborativa; se reafirma que es en sociedad en donde se consigue el aprendizaje y que el docente se convierte en mediador del mismo.

El constructivismo social se basa en la interacción de la persona con su medio, con los demás; el contexto es considerado como el lugar en el cual suceden las interacciones, es por ello, que al hablar de contexto se refiere a la interacción social, en la cual incide la relación con el entorno en general (Gonzáles, 2012). No es posible el aprendizaje para una persona, si no está ligada íntimamente al contexto en el que vive, lo cual va acorde con que el apropiarse del conocimiento es una construcción social e individual que parte de la interacción con el medio. En todo caso, todo conocimiento se 
construye a partir de la interacción de los todos los componentes tomando en consideración el medio en el que se desenvuelven y la realidad (Grajeda de Paz, 2001).

En relación a las competencias digitales, (Ferrari, Punie, \& Redecker, 2012) afirman que la competencia digital es la unión o suma de numerosas alfabetizaciones y la otra conceptualización corresponde a que la competencia digital es una nueva forma de alfabetización, pues implica nuevos retos y /o formas de alfabetizar.

El término competencia es relativamente joven, pues para considerar a alguien competente una persona debe desempeñarse en un contexto de manera autónoma, eficiente y crítica (Zahonero \& Bris, 2015)

Para (Imbernón, y otros, 2008) se considera al docente como competente cuando es capaz de resolver con coherencia una situación dentro de un contexto diferente con complejidad de por medio; además puede brindan una serie de posibilidad de resolución, considerando los diferentes resultados obtenidos en dicho proceso.

Según (Fink, 1995), un programa demanda un esfuerzo sistemático con la intención de lograr una serie de propósitos planificados. Dichos propósitos pueden ser muy ambiciosos o limitados, pueden darse en los diferentes ámbitos y espacios geográficos; así también, pueden desarrollarse a partir de estructuras y organizaciones variadas, así también, puede contar con propósitos y participantes diferentes. También es definida como el conglomerado de conocimientos, habilidades y actitudes indispensables en el momento actual para desempeñarse dentro de un entorno digital, por ende, el ser competente digital comprende la adquisición de una gran capacidad adaptativa acorde con las nuevas necesidades que surgen tanto en el ámbito tecnológico como en el personal; con la finalidad de interactuar eficientemente en torno a ellas (IKANOS, 2016). Asimismo, teniendo como base este proyecto DIGCOMP, se identifican cinco dimensiones actualizadas al año 2017: información y alfabetización informacional, comunicación y colaboración, creación de contenido digital, seguridad y resolución de problemas.

En cuanto a la información y alfabetización informacional que consiste en poder identificar, ubicar, recuperar, guardar, organizar y tener la capacidad de analizar la información digital, considerando la finalidad y trascendencia; mientras la comunicación y colaboración refiere la comunicación mediante entornos virtuales, el compartir recursos en línea, interactuar y participar en comunidades digitales; sobre la creación de contenido digital, comprende el elaborar y editar nuevos contenidos, ya sean imágenes, textos, producciones artísticas, contenidos multimedia y programación informática, aplicando los derechos de propiedad intelectual y licencias de uso; con 
respecto a la dimensión seguridad, se refiere a la protección personal y de datos, de la identidad digital y uso de seguridad cuando se navegue por la red y por último, sobre la dimensión resolución de problemas, se refiere a identificar las necesidades y recursos digitales, la selección de la herramienta digital indicada, de acuerdo a la finalidad o necesidad, el poder resolver diferentes problemas técnicos, el usar creativamente la tecnología y mantenerse actualizado (IKANOS, 2016).

Dentro de este marco, se estructura un programa de intervención es la provisión inteligente de las etapas del trabajo y la programación de las actividades de tal manera que la enseñanza se dé considerando, la seguridad, economía y eficiencia. Asi, es un plan ordenado donde se ejecutan acciones necesarias para conseguir el objetivo propuesto. 


\section{Método}

Se realizó una investigación aplicada, cuyo objetivo es evaluar los efectos del programa de intervención para resolver un determinado problema. Para (Sánchez F. A., 2019) la investigación aplicada su característica principal radica en la búsqueda de la aplicación de los conocimientos almacenados, con la intención de adquirir otros nuevos, después de implementar y sistematizar la práctica basada en la investigación. Este tipo de investigación permite conocer la realidad gracias al uso de conocimientos y la obtención de resultados.

Según su enfoque, la presente investigación es de tipo cuantitativa, pues medirá la relación entre las variables con datos numéricos, haciendo uso de la estadística. El enfoque cuantitativo representa un conjunto de procesos, tiene una secuencia y busca probar o comprobar hipótesis; cada una de las etapas del proceso de investigación precede a la anterior, lo que significa que el orden es riguroso (Hernández, Fernández, \& Baptista, 2014). Una investigación cuantitativa parte de una idea que una vez delimitada, permite la generación de objetivos y preguntas de investigación, se analiza la literatura al respecto y se elabora un marco teórico. A partir de las preguntas se elaboran hipótesis y variables con la intención de probarlas, para ello, se miden en un determinado contexto, se hace uso de la estadística para poder realizar mediciones y se extraen conclusiones (Hernández, Fernández, \& Baptista, 2014), para poder determinar los efectos del programa de intervención en el desarrollo de la competencia digital de los docentes donde se analizaron y evaluaron los resultados obtenidos de la variable dependiente a través de un pretest y postest.

El diseño fue experimental requiere de la manipulación intencional de acciones para poder analizar los posibles resultados. Los experimentos se encargan de la manipulación de tratamientos, estímulos, influencias o intervenciones para observar sus efectos sobre otras variables. El presente estudio se considera un grupo experimental conformado por docentes de educación básica regular a las cuales se les aplicó cuatro tratamientos con pretest y postest, como se muestra en la imagen

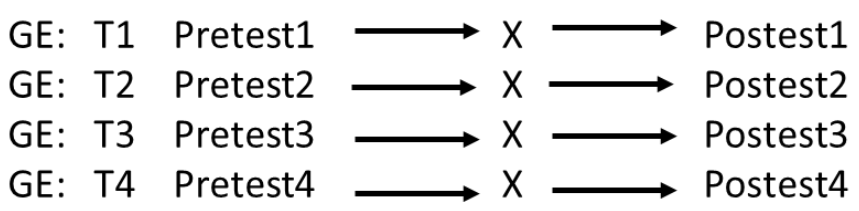

Figura 1. Tratamientos experimentales

Donde: 
$\begin{array}{lll}\text { GE } & : & \text { Grupo experimental } \\ \mathrm{X} & : & \text { Variable independiente Programa de Intervención }\end{array}$

Se operacionalizó la variable a través de la descomposición de la variable dependiente en dimensiones y éstas en indicadores para generar reactivos y obtener los datos (Córdova, 2018).

Tabla 1:

Operacionalización de la variable dependiente: Competencia digital docente

\begin{tabular}{|c|c|c|c|c|}
\hline Dimensiones & Indicadores & Ítems & Escalas & $\begin{array}{c}\text { Niveles y } \\
\text { Rango }\end{array}$ \\
\hline $\begin{array}{l}\text { Información y } \\
\text { alfabetización } \\
\text { informacional }\end{array}$ & $\begin{array}{l}\text { Navega, busca y } \\
\text { filtra información. } \\
\text { Almacena y recupera } \\
\text { información }\end{array}$ & $1-2-3$ & \multirow{5}{*}{$\begin{array}{l}1=\text { nunca } \\
2=\text { casi nunca } \\
3=\text { a veces } \\
4=\text { casi siempre } \\
5=\text { siempre }\end{array}$} & \multirow{5}{*}{$\begin{array}{l}\text { Adecuadas } \\
(77-100) \\
\text { Poco } \\
\text { adecuado } \\
(41-76) \\
\text { Inadecuada } \\
(20-40)\end{array}$} \\
\hline $\begin{array}{l}\text { Comunicación y } \\
\text { colaboración }\end{array}$ & $\begin{array}{l}\text { Comparte } \\
\text { información y } \\
\text { contenidos digitales. } \\
\text { Participa en línea. } \\
\text { Colabora a través de } \\
\text { canales digitales. }\end{array}$ & $4-5-6$ & & \\
\hline $\begin{array}{l}\text { Creación de } \\
\text { contenido digital }\end{array}$ & $\begin{array}{l}\text { Crea material digital } \\
\text { haciendo } \\
\text { Elabora contenido } \\
\text { digital. }\end{array}$ & $7-8-9$ & & \\
\hline Seguridad & $\begin{array}{l}\text { Utiliza los medios de } \\
\text { seguridad para } \\
\text { resguardar su } \\
\text { información. } \\
\text { Publica información } \\
\text { manteniendo límites }\end{array}$ & $10-11-12$ & & \\
\hline $\begin{array}{l}\text { Resolución de } \\
\text { problemas }\end{array}$ & $\begin{array}{l}\text { Utiliza la tecnología } \\
\text { creativamente- Elije } \\
\text { herramientas } \\
\text { digitales apropiadas } \\
\text { para el trabajo. }\end{array}$ & $13-14-15$ & & \\
\hline
\end{tabular}

Población conformada por docentes de una institución educativa, siendo la cantidad total del fenómeno a ser estudiado que tienen alguna característica en común y origina la investigación (Arias-Gómez, Villasís-Keever, \& Novales, 2016).

La técnica para la recolección de información y datos que se empleó fue la encuesta, que según (Sánchez \& Reyes, 2017) es una técnica de investigación usada para la indagación, exploración y recolección de datos, para ello, hace uso de preguntas 
formuladas ya sea de forma directa o indirecta a las personas que forman parte de la unidad de estudio para la investigación.

La confiabilidad del instrumento permite la congruencia de las medidas que permiten resolver los problemas (Kerlinger, 2002) donde de acuerdo al Alpha de Cronbach fue de 0.942 , es decir altamente confiable. Asimismo, la validez de acuerdo a (Magnusson, 1978) mide lo que desea medir, donde este fue validado por cinco expertos. Con respecto al procedimiento se aplicó un programa estadístico que permitió realizar la descripción y el análisis inferencial se empleó Anova para un factor.

\section{Resultados}

El análisis descriptivo, luego de la aplicación antes y después de cada tratamiento del programa de intervención con relación a la variable competencia digital muestran que el tratamiento que presentó mayores cambios fue el tratamiento 3, donde antes de la aplicación su media presenta 2,66 y después de la aplicación la media obtenida fue de 3,85 y además su desviación estándar fue de ,688. En cambio, el tratamiento que tuvo resultados menos satisfactorios fue el primero donde su media presenta 2,65 y después de la aplicación la media obtenida fue de 3,50 y además su desviación estándar fue de ,852.

\section{Tabla 1}

Comparación de tratamientos del programa de intervención

\begin{tabular}{llllc}
\hline Tratamiento & & Media & $\mathrm{N}$ & Desviación típ. \\
\hline \multirow{2}{*}{ Tratamiento 1 } & Tratamiento 1 (Antes) & 2,65 & 24 &, 944 \\
& Tratamiento 1 (Después) & 3,50 & 24 &, 852 \\
\cline { 2 - 5 } Tratamiento 2 & Tratamiento 2 (Antes) & 3,05 & 24 &, 845 \\
& Tratamiento 2 (Después) & 3,59 & 24 &, 819 \\
Tratamiento 3 & Tratamiento 3 (Antes) & 2,66 & 24 &, 927 \\
\multirow{2}{*}{ Tratamiento 4 } & Tratamiento 3 (Después) & 3,85 & 24 &, 688 \\
\cline { 2 - 5 } & Tratamiento 4 (Antes) & 2,91 & 24 &, 845 \\
& Tratamiento 4 (Después) & 3,55 & 24 &, 706
\end{tabular}




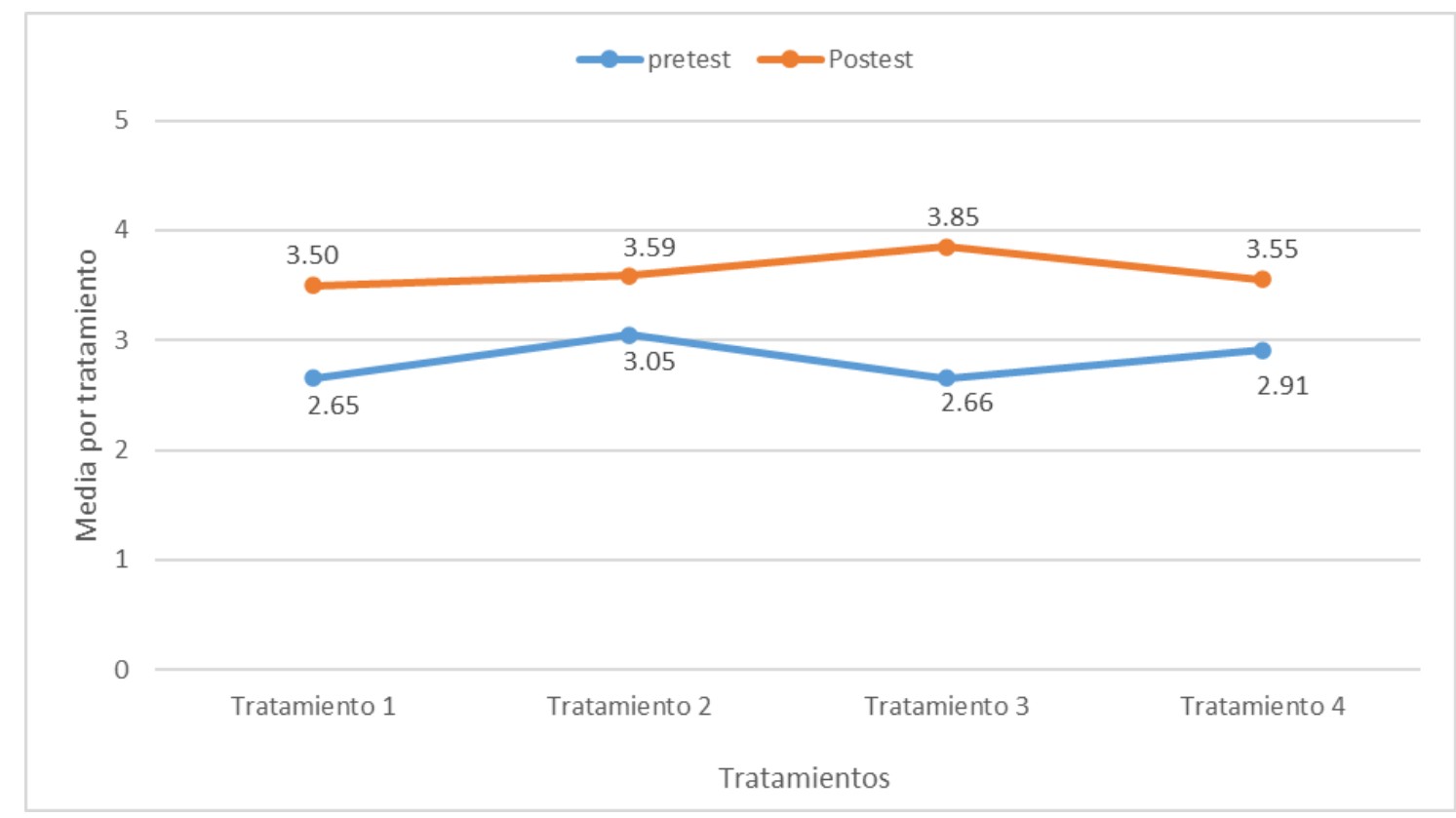

Figura 1. Comparación de tratamientos del programa de intervención.

En relación a la dimensión información y alfabetización, que el tratamiento que presentó mayores cambios fue el tratamiento 4, donde antes de la aplicación su media presenta 2,9858 y después de la aplicación la media obtenida fue de 4,0279 y además su desviación estándar fue de ,64468. En cambio, el tratamiento que tuvo resultados menos satisfactorios fue el 4 donde su media presenta 3,0271y después de la aplicación la media obtenida fue de 3,6388 y además su desviación estándar fue de 63601 .

Tabla 2

Comparación de tratamientos del programa de intervención, dimensión Información y alfabetización informacional

\begin{tabular}{|c|c|c|c|c|c|c|c|c|}
\hline & & \multirow[t]{2}{*}{$\mathrm{N}$} & \multirow[t]{2}{*}{ Media } & \multirow{2}{*}{$\begin{array}{l}\text { Desviación } \\
\text { típica }\end{array}$} & \multicolumn{2}{|c|}{$\begin{array}{c}\text { Intervalo de } \\
\text { confianza para la } \\
\text { media al } 95 \%\end{array}$} & \multirow[t]{2}{*}{ Mínimo } & \multirow[t]{2}{*}{ Máximo } \\
\hline & & & & & $\begin{array}{l}\text { Límite } \\
\text { inferior }\end{array}$ & $\begin{array}{l}\text { Límite } \\
\text { superior }\end{array}$ & & \\
\hline D1 Pre & Tratamiento 1 & 24 & 3,0554 & 1,04715 & 2,6132 & 3,4976 & 1,00 & 5,00 \\
\hline \multirow[t]{3}{*}{ test } & Tratamiento 2 & 24 & 3,2925 & ,95523 & 2,8891 & 3,6959 & 1,67 & 5,00 \\
\hline & Tratamiento 3 & 24 & 2,9858 & 1,00961 & 2,5595 & 3,4122 & 1,00 & 5,00 \\
\hline & Tratamiento 4 & 24 & 3,0271 & ,91156 & 2,6422 & 3,4120 & 1,00 & 4,67 \\
\hline $\mathrm{D} 1$ & Tratamiento 1 & 24 & 3,9171 & ,97919 & 3,5036 & 4,3306 & 1,67 & 5,00 \\
\hline \multirow[t]{3}{*}{ Postest } & Tratamiento 2 & 24 & 3,6521 & ,72497 & 3,3460 & 3,9582 & 1,67 & 4,67 \\
\hline & Tratamiento 3 & 24 & 4,0279 & ,64468 & 3,7557 & 4,3001 & 3,00 & 5,00 \\
\hline & Tratamiento 4 & 24 & 3,6388 & ,63601 & 3,3702 & 3,9073 & 2,67 & 5,00 \\
\hline
\end{tabular}


Los resultados de la dimensión comunicación y colaboración muestran que el tratamiento que presentó mayores cambios fue el tratamiento 4, donde antes de la aplicación su media presenta 3,0838 y después de la aplicación la media obtenida fue de 3,7925 y además su desviación estándar fue de ,77260. En cambio, el tratamiento que tuvo resultados menos satisfactorios fue el 3 donde su media presenta 2,4854 y después de la aplicación la media obtenida fue de 3,7500 y además su desviación estándar fue de ,87449.

Tabla 3

Comparación de tratamientos del programa de intervención, dimensión Comunicación y colaboración

\begin{tabular}{|c|c|c|c|c|c|c|c|c|}
\hline & & \multirow{2}{*}{$\mathrm{N}$} & \multirow{2}{*}{ Media } & \multirow{2}{*}{$\begin{array}{l}\text { Desviación } \\
\text { típica }\end{array}$} & \multicolumn{2}{|c|}{$\begin{array}{c}\text { Intervalo de } \\
\text { confianza para la } \\
\text { media al } 95 \%\end{array}$} & \multirow[t]{2}{*}{ Mínimo } & \multirow[t]{2}{*}{ Máximo } \\
\hline & & & & & $\begin{array}{l}\text { Límite } \\
\text { inferior }\end{array}$ & $\begin{array}{c}\text { Límite } \\
\text { superior }\end{array}$ & & \\
\hline D2 Pre & Tratamiento 1 & 24 & 3,3621 & 1,04969 & 2,9188 & 3,8053 & 1,00 & 4,67 \\
\hline \multirow[t]{3}{*}{ test } & Tratamiento 2 & 24 & 3,1800 & 99788 & 2,7586 & 3,6014 & 1,67 & 5,00 \\
\hline & Tratamiento 3 & 24 & 2,4854 & 1,07700 & 2,0306 & 2,9402 & 1,00 & 5,00 \\
\hline & Tratamiento 4 & 24 & 3,0838 & 1,06505 & 2,6340 & 3,5335 & 1,00 & 5,00 \\
\hline D2 & Tratamiento 1 & 24 & 3,8471 & ,68133 & 3,5594 & 4,1348 & 2,33 & 5,00 \\
\hline \multirow[t]{3}{*}{ Postest } & Tratamiento 2 & 24 & 3,8067 & ,94232 & 3,4088 & 4,2046 & 1,67 & 5,00 \\
\hline & Tratamiento 3 & 24 & 3,7500 & 87449 & 3,3807 & 4,1193 & 2,00 & 5,00 \\
\hline & Tratamiento 4 & 24 & 3,7925 & ,77260 & 3,4663 & 4,1187 & 2,67 & 5,00 \\
\hline
\end{tabular}

Los resultados de la dimensión creación de contenido digital muestran que el tratamiento que presentó mayores cambios fue el tratamiento 3 , donde antes de la aplicación su media presenta 2,5279 y después de la aplicación la media obtenida fue de 3,8346 y además su desviación estándar fue de ,79883. En cambio, el tratamiento que tuvo resultados menos satisfactorios fue el 4 donde su media presenta 3,3467 y después de la aplicación la media obtenida fue de 2,7783 y además su desviación estándar fue de ,88799. 
Tabla 4

Comparación de tratamientos del programa de intervención, dimensión creación de contenido digital

\begin{tabular}{|c|c|c|c|c|c|c|c|c|}
\hline & & $\mathrm{N}$ & Media & Desviació & $\begin{array}{r}\text { Inter } \\
\text { confian } \\
\text { media }\end{array}$ & $\begin{array}{l}\text { alo de } \\
\text { a para la } \\
\text { al } 95 \%\end{array}$ & Mínimo & Máximo \\
\hline & & & & & $\begin{array}{l}\text { Límite } \\
\text { inferior }\end{array}$ & $\begin{array}{l}\text { Límite } \\
\text { superior }\end{array}$ & & \\
\hline D3 Pre & Tratamiento 1 & 24 & 2,8892 & 1,18225 & 2,3899 & 3,3884 & 1,00 & 5,00 \\
\hline test & Tratamiento 2 & 24 & 3,1388 & 97788 & 2,7258 & 3,5517 & 1,00 & 5,00 \\
\hline & Tratamiento 3 & 24 & 2,5279 & 1,02595 & 2,0947 & 2,9611 & 1,00 & 5,00 \\
\hline & Tratamiento 4 & 24 & 2,7783 & 1,01479 & 2,3498 & 3,2068 & 1,33 & 5,00 \\
\hline D3 & Tratamiento 1 & 24 & 3,8196 & ,97790 & 3,4067 & 4,2325 & 1,67 & 5,00 \\
\hline Postest & Tratamiento 2 & 24 & 3,5967 & ,70136 & 3,3005 & 3,8928 & 1,67 & 4,67 \\
\hline & Tratamiento 3 & 24 & 3,8346 & ,79883 & 3,4973 & 4,1719 & 2,00 & 5,00 \\
\hline & Tratamiento 4 & 24 & 3,3467 & ,88799 & 2,9717 & 3,7216 & 2,00 & 5,00 \\
\hline
\end{tabular}

Los resultados de la dimensión seguridad muestran que el tratamiento que presentó mayores cambios fue el tratamiento 3, donde antes de la aplicación su media presenta 2,3071 y después de la aplicación la media obtenida fue de 3,7225 y además su desviación estándar fue de ,81492. En cambio, el tratamiento que tuvo resultados menos satisfactorios fue el 4 donde su media presenta 1,7771 y después de la aplicación la media obtenida fue de 2,7079 y además su desviación estándar fue de 1,20521.

Tabla 5

Comparación de tratamientos del programa de intervención, dimensión aplicación del programa CODI, dimensión seguridad

\begin{tabular}{|c|c|c|c|c|c|c|c|c|}
\hline & & \multirow[t]{2}{*}{$\mathrm{N}$} & \multirow[t]{2}{*}{ Media } & \multirow{2}{*}{$\begin{array}{c}\text { Desviació } \\
\text { n típica }\end{array}$} & \multicolumn{2}{|c|}{$\begin{array}{c}\text { Intervalo de } \\
\text { confianza para la } \\
\text { media al } 95 \%\end{array}$} & \multirow[t]{2}{*}{ Mínimo } & \multirow[t]{2}{*}{ Máximo } \\
\hline & & & & & $\begin{array}{l}\text { Límite } \\
\text { inferior }\end{array}$ & $\begin{array}{c}\text { Límite } \\
\text { superior }\end{array}$ & & \\
\hline D4 Pre & Tratamiento 1 & 24 & 1,7771 & ,96105 & 1,3713 & 2,1829 & 1,00 & 4,33 \\
\hline \multirow[t]{3}{*}{ test } & Tratamiento 2 & 24 & 2,6521 & 88682 & 2,2776 & 3,0266 & 1,00 & 4,33 \\
\hline & Tratamiento 3 & 24 & 2,3071 & 99766 & 1,8858 & 2,7284 & 1,00 & 4,00 \\
\hline & Tratamiento 4 & 24 & 2,9442 & ,97680 & 2,5317 & 3,3566 & 1,00 & 4,67 \\
\hline D4 & Tratamiento 1 & 24 & 2,7079 & 1,20521 & 2,1990 & 3,2168 & 1,00 & 5,00 \\
\hline \multirow[t]{3}{*}{ Postest } & Tratamiento 2 & 24 & 3,2913 & 1,03713 & 2,8533 & 3,7292 & 1,00 & 5,00 \\
\hline & Tratamiento 3 & 24 & 3,7225 & 81492 & 3,3784 & 4,0666 & 2,33 & 5,00 \\
\hline & Tratamiento 4 & 24 & 3,6250 & 84775 & 3,2670 & 3,9830 & 2,00 & 5,00 \\
\hline
\end{tabular}


Los resultados de la dimensión resolución de problemas muestran que el tratamiento que presentó mayores cambios fue el tratamiento 3, donde antes de la aplicación su media presenta 2,9725 y después de la aplicación la media obtenida fue de 3,9025 y además su desviación estándar fue de ,71932. En cambio, el tratamiento que tuvo resultados menos satisfactorios fue el 1 donde su media presenta 2,1808 y después de la aplicación la media obtenida fue de 3,1937 y además su desviación estándar fue de 1,18422.

Tabla 6

Comparación de tratamientos del programa de intervención, dimensión resolución de problemas

\begin{tabular}{|c|c|c|c|c|c|c|c|c|}
\hline & & \multirow[t]{2}{*}{$\mathrm{N}$} & \multirow[t]{2}{*}{ Media } & \multirow{2}{*}{$\begin{array}{l}\text { Desviación } \\
\text { típica }\end{array}$} & \multicolumn{2}{|c|}{$\begin{array}{c}\text { Intervalo de } \\
\text { confianza para la } \\
\text { media al } 95 \%\end{array}$} & \multirow[t]{2}{*}{ Mínimo } & \multirow[t]{2}{*}{ Máximo } \\
\hline & & & & & $\begin{array}{l}\text { Límite } \\
\text { inferior }\end{array}$ & $\begin{array}{c}\text { Límite } \\
\text { superior }\end{array}$ & & \\
\hline D5 Pre & Tratamiento 1 & 24 & 2,1808 & 1,06775 & 1,7300 & 2,6317 & 1,00 & 5,00 \\
\hline \multirow[t]{3}{*}{ test } & Tratamiento 2 & 24 & 2,9867 & 1,01502 & 2,5581 & 3,4153 & 1,33 & 5,00 \\
\hline & Tratamiento 3 & 24 & 2,9725 & ,93131 & 2,5792 & 3,3658 & 1,00 & 5,00 \\
\hline & Tratamiento 4 & 24 & 2,7217 & 86138 & 2,3579 & 3,0854 & 1,33 & 4,33 \\
\hline D5 & Tratamiento 1 & 24 & 3,1937 & 1,18422 & 2,6937 & 3,6938 & 1,00 & 5,00 \\
\hline \multirow[t]{3}{*}{ Postest } & Tratamiento 2 & 24 & 3,5975 & 99726 & 3,1764 & 4,0186 & 1,67 & 5,00 \\
\hline & Tratamiento 3 & 24 & 3,9025 & ,71932 & 3,5988 & 4,2062 & 2,33 & 5,00 \\
\hline & Tratamiento 4 & 24 & 3,3471 & 86488 & 2,9819 & 3,7123 & 1,67 & 5,00 \\
\hline
\end{tabular}

\section{Contrastación de hipótesis}

\section{Sistemas de Hipótesis de la investigación}

$\mathrm{H}_{0}$ : La aplicación del programa intervención no presenta efectos positivos en el desarrollo de las competencias digitales y sus dimensiones de docentes de nuna institución educativa pública.

Ho: $\mu_{\text {antes }}=\mu_{\text {después }}$

$\mathrm{H}_{1}$ : La aplicación del programa intervención presenta efectos positivos en el desarrollo de las competencias digitales y sus dimensiones de docentes de nuna institución educativa pública.

Hi: $\mu a_{n t e s}<\mu_{\text {después }}$

La Prueba de Levene nos indica que el p-valor es mayor que 0.05, aceptándose la hipótesis de similitud de varianzas, concluyéndose que las poblaciones de la variable en estudio son similares, para todos los casos. Asimismo, de acuerdo a la hipótesis 
planteada, se muestran los índices de F= ,981 y p-valor ,405; que nos indican que los procesos de aprendizaje se han realizado satisfactoriamente en los tratamientos empleados para el desarrollo de competencias digitales.

Tabla 7

Prueba de homogeneidad de varianzas de la variable competencias digitales

\begin{tabular}{|c|c|c|c|c|c|c|c|}
\hline \multicolumn{6}{|c|}{ Prueba de homogeneidad de varianzas } & \multicolumn{2}{|c|}{ Anova } \\
\hline & & $\begin{array}{l}\text { Estadístico } \\
\text { de Levene }\end{array}$ & gl1 & gl2 & Sig. & $F$ & Sig. \\
\hline \multirow{4}{*}{$\begin{array}{l}\text { Competencias } \\
\text { digitales } \\
\text { (postest) }\end{array}$} & Se basa en la media & ,605 & 3 & 92 & ,614 & 5,146 & 0,002 \\
\hline & Se basa en la mediana &, 527 & 3 & 92 & ,665 & & \\
\hline & $\begin{array}{l}\text { Se basa en la mediana y } \\
\text { con gl ajustado }\end{array}$ &, 527 & 3 & $\begin{array}{r}85,73 \\
0\end{array}$ & ,665 & & \\
\hline & $\begin{array}{l}\text { Se basa en la media } \\
\text { recortada }\end{array}$ & ,577 & 3 & 92 & ,632 & & \\
\hline
\end{tabular}

Con referencia a las hipótesis específicas, en concordancia con las dimensiones de las variables, nos demuestran resultados similares, por lo que se concluye que los el programa de intervención resulto satisfactorio.

\section{Discusión}

El tratamiento de la información y el análisis estadístico, en referencia a la hipótesis general, muestran los valores medios de la aplicación del programa intervención en los cuatro tratamientos, donde las medias evolucionaron satisfactoriamente. Asimismo, el estadístico anova nos indica que el proceso de aprendizaje fue producto del programa de intervención.

Estos hallazgos relevantes son concordantes con (Ojeda, Reyes, \& Reyes, 2018) donde a través programa de alfabetización digital para profesores de escuelas normales tuvieron el éxito deseado al igual que (López, 2018) con un programa de actualización docente en las TIC, cuyo propósito fue encontrar las carencias instructivas en la utilización de las TIC, lograron actualizar los docentes del nivel inicial y primaria en dichas herramientas tecnológicas.

Las coincidencias y los hallazgos muestran que el conectivismo de Siemens, es concordante con las nuevas tecnologías para su aplicación y descripción. El análisis permite a este modelo darle una estructura, donde se requiere de ciertas habilidades de aprendizaje, pero en el mundo digital, cuya actividad es colectiva donde las redes permiten el intercambio fluido de conocimientos y el trabajo cooperativo. De allí la importancia de que el docente debe estar en permanente preparación. 
Es así, que el conectivismo basado en las habilidades de aprendizaje y en el uso de las herramientas informáticas, han construido aprendizajes, gracias a los procesos aprendizaje-enseñanza donde la función del docente es de acompañamiento, guía, conducción, tutorización del trabajo en redes.

Los resultados referentes a las dimensiones de las variables (hipótesis especificas) son concordantes con (Hernández, Gámiz, \& Romero, 2020) con su programa para transformar la competencia digital en docentes universitarios de México y España, donde ha permitido el entendimiento de la competencia digital, como un suplemento para planes e instrucción del profesor. Esto nos demuestra que las competencias digitales permiten darle relevancia en la construcción del conocimiento gracias al intercambio permanente entre estudiantes y docentes siendo cada uno los protagonistas de este espacio, y de esta forma de enseñanza aprendizaje se obtienen resultados satisfactorios. Por ello (Piaget, 1978) afirma que el proceso de alojamiento es la experiencia directa y asimilación de la información que son fundamentales, tanto como la experiencia directa.

En relación al trabajo de (Chou, Valdés, \& Sánchez, 2017) con el programa para el desarrollo de las TIC, donde el resultado nos indicó que se convirtió en un eficaz instrumento que propicia aprendizaje, asegurando la innovación de este proceso. Para (Pérez, Jordano de la Torre, \& Martín-Cuadrado, 2017) con la aplicación de curso Nano Curso masivo en Abierto que se convirtió en herramienta básica para la adquisición de aprendizajes.

Esto, desde la perspectiva de Siemens, donde el aprendizaje y el conocimiento es definido desde diferentes perspectivas, diversas fuentes de información, na convirtiéndose en medio para aprender y haciendo uso de la tecnología permitiéndose para descubrir y aprender cuando hay conexiones manteniendo el conocimiento actualizado y contextualizado.

Con referencia a (Kenneth, 2012) al aplicarse los principios de redes permite definir el conocimiento como el mismo proceso de aprendizaje. Complementariamente la teoría constructivista y el constructivismo social (Ortega \& Gacitúa, 2008) permite que la interacción social este potenciada a través del uso de los medios tecnológicos, para el aprendizaje y en equipo.

Cuando nos referimos al tratamiento de la información, los estudios de (Guizado, Menacho, \& Salvatierra, 2019; Lamacponga, 2019; Hatlevik, 2017) establecieron la relación entre competencia digital y el desarrollo profesional docente, determinando que 
que el uso de entorno virtual de aprendizaje está relacionado con el desarrollo de las competencias digitales.

Es importante destacar lo afirmado por (Hernández, Gámiz, \& Romero, 2020) donde establecen los referentes al constructivismo, la interacción de las personas o estudiantes con el medio real, con el contexto o la situación y las personas con un elemento activo dentro del medio en el que se encuentren, pues brindan significado con sus aportaciones, ideas, pensamientos, como también, reciben las ideas, representaciones y pensamientos de las otras personas que conforman el medio de intercambio. Es aquí donde la investigación sienta sus bases en la teoría socio cultural de Vygotsky, porque el medio social es fundamental para el desarrollo de la persona, pues la interacción social, haciendo uso de herramientas es lo que permite el aprendizaje.

Es importante explicar que estudios realizados por (Lores, Sánchez, \& García, 2019) al realizar los estudios sobre las competencias digitales demuestra que debido a la preparación puramente teórica los docentes estos tienen mala formación en la competencia digital. Por ello, desde la perspectiva sociocultural que tiene una visión del aprendizaje en la que el entorno social y la colaboración mediada por el lenguaje como herramienta psicológica juegan un papel fundamental para el logro de objetivos y en donde las actividades humanas ocurren en sociedad y por ellos no se pueden comprender fuera de ellas, destacando la importancia del trabajo cooperativo y la necesidad de la preparación continua, permanente de los docentes en su proceso formativo.

De allí, que las competencias digitales influyen en las competencias pedagógicas, se deben aplicar en los procesos educativos y administrativos, mostrándose una necesidad de formar personas competentes digitales. Se debe adquirir conocimientos de forma personal e interpersonal, generado por las relaciones sociales con las diferentes personas de su entorno y ello permite que sea un conocimiento más enriquecido y productivo.

El análisis de la información nos lleva a definir el termino de competencia, como producto de los aportes de los investigadores: el uso seguro y crítico de las Tics con el propósito de trabajar, descansar o comunicarse y que debe desempeñarse en un contexto de manera autónoma, eficiente y crítica para la resolución de problemas (Ferrari, Punie, \& Redecker, 2012; Zahonero \& Bris, 2015; Imbernón, y otros, 2008)

Asimismo, el programa aplicado como conjunto de actividades realizado tuvo la intención de que cada persona cumpla con su formación completa según lo esperado, 
demostrando su provisión inteligente en todas las etapas del trabajo de las actividades programadas dando la seguridad, economía y eficiencia. Por ello, busco que los docentes se involucren en la tecnología digital y puedan desarrollar sus clases a través de plataformas o haciendo uso de las diferentes herramientas digitales. Por último, el programa tuvo las condiciones necesarias, amplio, completo, flexible, graduado, adecuado y realizable y que estuvo compuesto por objetivos, lo que se pretende alcanzar; los contenidos, para poder dosificarlos e ir impartiendo según necesidades y la evaluación, para medir si se logran los contenidos que se enseña.

\section{Conclusiones}

Se ha demostrado la efectividad del programa de intervención en el desarrollo de las competencias digitales con la aplicación de cuatro tratamientos en un diseño experimental señala el crecimiento del aprendizaje en los docentes involucrados. Esto nos lleva a que la realización de los procesos de formación continua de desarrollo de competencias, se deben realizar actividades donde se comprenda ese conjunto de cualidades, capacidades y aptitudes que permiten al docente a resolver sus problemas pedagógicos y que además permiten su constante aprendizaje.

Así, la dotación de herramientas no solo tecnológicas si no también mentales les permitirá gestionar el conocimiento, de tal manera que puedan actuar ante las diversas situaciones que se presentan, utilizar y combinar medios y recursos para su movilización en el aprendizaje, realizar la transferencia de conocimientos, para modelar el aprendizaje, aprender para seguir aprendiendo, y comprometerse, asumiendo los riesgos necesarios para innovar su experiencia didáctica.

Asimismo, desarrollar en los docentes dos tipos de competencias fundamentales, las básicas y las específicas. La primera referida al intelecto, comunicación, gestión, éticas, entre otras; las segunda referidas, al conocimiento, tecnologías y técnicas, profesionales y académicas. La formación del docente debe ser teórica-práctico, que les permita el afianzamiento y desarrollo de los aprendizajes y de las competencias a desarrollar, ejercitando permanentemente estas hasta llegar a nivel de habilidades.

\section{Referencias}

Area, M., \& Guarro, A. (2012). La alfabetización informacional y digital: fundamentos pedagógicos para la enseñanza y el aprendizaje 
competente. Revista Española De Documentación Científica(35), 46,74. doi:https://doi.org/10.3989/redc.2012.mono.977

Arias-Gómez, J., Villasís-Keever, M. Á., \& Novales, M. G. (2016). El protocolo de investigación III: la población de estudio . Revista Alergia México, 63(2), 201-206. Obtenido de [Fecha de consulta 22 de julio de 2020]. ISSN: 0002-5151.

Disponible

en:

https://www.redalyc.org/articulo.oa?id=4867/486755023011

Chou, D., Valdés, M., \& Sánchez, D. (2017). Programa de formación de competencias digitales en docentes universitarios. Universidad $y$ Sociedad, $9(1), \quad$ 81-86. Obtenido de https://rus.ucf.edu.cu/index.php/rus/article/view/518

Comunidades Europeas. (2007). Competencias clave para el aprendizaje permanente. Un Marco de Referencia Europeo. Obtenido de Recuperado de http://www.mecd.gob.es/dctm/ministerio/educacion/mecu/movilidadeuropa/competenciasclave.pdf?documentld=0901e72b80685fb1

Córdova, I. (2018). Instrumentos de investigación (1era edición ed.). Lima: Editorial San Marcos.

Ferrari, A., Punie, Y., \& Redecker, C. (2012). Understanding Digital Competence in the 21st Century: An Analysis of Current Frameworks. En Lecture Notes in Computer Science (págs. 79-92). Springer. doi:https://doi.org/10.1007/978-3-642-33263-0

Fink, A. (1995). Evaluation for education and psychology. California: Sage Publications.

Gonzáles, C. (2012). Aplicación del Constructivismo Social en el Aula. Obtenido de https://www.oei.es/historico/formaciondocente/materiales/OEl/2012_GO NZALEZ_ALVAREZ.pdf

Grajeda de Paz, M. (2001). Transformar la práctica educativa. Guatemala: Saqil Tzij.

Guizado, F., Menacho, I., \& Salvatierra, A. (2019). Competencia digital y desarrollo profesional de los docentes de dos instituciones de educación 
básica regular del distrito de Los Olivos, Lima-Perú. Humatay, 6(1). doi:http://dx.doi.org/10.21503/hamu.v6i1.1574

Hatlevik, O. (2017). Examining the Relationship between Teachers' Self-Efficacy, their Digital Competence, Strategies to Evaluate Information, and use of ICT at School. Scandinavian Journal of Educational Research, 61(5), 555.567. doi:10.1080/00313831.2016.1172501

Hernández, A., Gámiz, V., \& Romero, M. (2020). Evolución de la competencia digital docente del profesorado universitario: incidentes críticos a partir de $\begin{array}{lll}\text { relatos de vida. Educar, 56(1), 109-127. } & \text {. }\end{array}$ doi:https://doi.org/10.5565/rev/educar.1088

Hernández, S. (2008). El modelo constructivista con las nuevas tecnologías: aplicado en el proceso de aprendizaje. Comunicación y construcción del conocimiento en el nuevo espacio tecnológico. Revista de Universidad y Sociedad del Conocimiento.

Hernández, S., Fernández, C., \& Baptista, L. (2014). Metodología de la Investigación. (6ta ed.). Caracas: MrGraw-Hill.

IKANOS. (2016). Obtenido de Euskadi.eus: https://www.innova.euskadi.eus/informacion/el-proyecto-digcomp/v62ikanoscd/es/

Imbernón, F., Carnicero, P., Silva, P., Cruz, L., Prats, M., Guzmán, C., \& Gonzáles, J. (2008). Análisis y propuestas de competencias docentes universitarias para el desarrollo del aprendizaje significativo del alumnado a través del e-learning y el b-learning en el marco del EEES. Barcelona: Ministerio de Educación y Ciencia. Programa de Estudio y Análisis.

Juca, F. (2016). La educación a distancia, una necesidad para la formación de los profesionales. Revista Universidad y Sociedad, 8(1), 106-111. Obtenido de http://scielo.sld.cu/pdf/rus/v8n1/rus15116.pdf

Kenneth, S. (2012). (Aprendizaje expansivo y conectivismo. Unoiversidad Continental.

Obtenido

de http://www.universidad.continental.edu.pe/Portal/wpcontent/uploads/doc umentos/revista_yachayninchik/08- 
Kerlinger, F. (2002). Investigación del comportamiento: Métodos de Investigación en Ciencias Sociales. México: McGraw Hill.

Lamacponga, Y. (2019). Entornos virtuales de aprendizaje y desarrollo de competencias digitales en los docentes. Yachay - Revista Científico Cultural, 7(1), 411-416. doi:https://doi.org/10.36881/yachay.v7i01.93

López, J. (2018). Estudio de un caso de la formación del profesorado en las tecnologías de la información y la comunicación en los centros de educación infantil y primaria. Didáctica, innovación y multimedia. Obtenido de http://dimglobal.net/revistaDIM36/DIMOC36formacionprofesorado.htm

Lores, B., Sánchez, P., \& García, M. (2019). La formación de la competencia digital en los docentes. Profesorado, Revista de Currículum y Formación del Profesorado, 23(4). Obtenido de https://revistaseug.ugr.es/index.php/profesorado/article/view/11720

Magnusson, D. (1978). Teoría de los Test. México: Editorial Trillas.

Martínez, S., El Berdai, M., \& Ortiz, A. (2017). Valoración del profesorado de educación secundaria de la ciudad de Tetuán sobre la formación en TIC desarrollada desde el Ministerio de Educación Nacional. Píxel-Bit. Revista de Medios y Educación(50). Obtenido de https://idus.us.es/handle/11441/51870

Moral, D., \& Villalustre, L. (2010). Formación del profesor 2.0: Desarrollo de competencias tecnológicas para la escuela. Revista Miscelania de investigación(23), 59-70. Obtenido de https://dialnet.unirioja.es/servlet/articulo?codigo $=3403432$

Ojeda, G., Reyes, G., \& Reyes, H. (2018). Programa de alfabetización digital para formadores de docentes. RECIE. Revista Electrónica Científica de Investigación Educativa, 691-708. Obtenido de https://www.rediech.org/ojs/2017/index.php/recie/issue/view/25

Ortega, J., \& Gacitúa, J. (2008). Espacios interactivos de comunicación y aprendizaje. La construcción de identidades. RUSC. Universities and Knowledge Society Journal, $5(2)$. doi:http://dx.doi.org/10.7238/rusc.v5i2.334 
Pérez, L., Jordano de la Torre, M., \& Martín-Cuadrado, A. (2017). Los NOOC para la formación en competencias digitales del docente universitario. Una experiencia piloto de la Universidad Nacional de Educación a distancia (UNED). Revista De Educación a Distancia (RED), 17(55). Obtenido de https://revistas.um.es/red/article/view/315281

Piaget, J. (1978). La representación del mundo en el niño. Madrid: Morata.

RAE. (2006). Diccionario de la Lengua Española .

Sánchez, F. A. (2019). Fundamentos epistémicos de la investigación cualitativa y cuantitativa: Consensos y disensos. Revista Digital de Investigación en Docencia Universitaria, 13(1), 102-122. doi:http://dx.doi.org/10.19083/ridu.2019.644

Sánchez, H., \& Reyes, C. (2017). Metodología y diseños en la investigación científica (Quinta ed.). Lima, Perú: Mantaro.

Siemens, G. (2004). Conectivismo: Una teoría de aprendizaje para la era digital. Obtenido

de https://www.comenius.cl/recursos/virtual/minsal_v2/Modulo_1/Recursos/ Lectura/conectivismo_Siemens.pdf

Zahonero, A., \& Bris, M. (2015). Formación integral del profesorado: Hacia el desarrollo de competencias personales y de valores en los docente. Tendencias pedagógicas, 20, 51-70. Obtenido de https://revistas.uam.es/tendenciaspedagogicas/article/view/2014 